\title{
Design of Optimized Mesh Network Routing Protocol Based on AODC
}

\author{
Xin Chen ${ }^{a}$, Aimin Cheng, Gengyu Ge, Yinmei Liu \\ Department of Information Engineering, Zunyi Normal College, Zunyi, Guizhou, China \\ achenxinza@foxmail.com
}

\begin{abstract}
Mesh network is a relatively new wireless network, which has the characteristics of large capacity, fast speed and huge space for development. This paper proposes an AODC_Improve routing protocol by optimizing the existing on-demand routing protocol (AODC). This protocol has the characteristics of end-to-end time delay and packet loss rate. There is a lot of optimization.
\end{abstract}

Keywords: AODC_Improve, optimization, mesh network, routing protocol.

\section{Introduction}

Wireless network is indispensable in our daily life, but at the same time, it is also faced with many problems, such as frequent web pages cannot be opened, slow download speed and so on. In the final analysis, there are still some problems in the wireless network itself. At present, we use AODC routing protocol in mesh network. In this network routing protocol, the network endpoint does not need to broadcast the information content in the network regularly, because when a network endpoint has data information to transmit, it will find the routing information itself, if it finds the routing information, it will find the routing information. Data will be transmitted according to the information found. However, there are some defects in the use of this protocol, that is, the relevant methods of node delay statistics are not accurate. Based on this, this paper proposes an AODC_Improve routing protocol to improve the use of mesh networks.

\section{Overview of Mesh Network Routing Protocol}

\subsection{The Basic Structure of Mesh Network}

AODC_Improve routing protocol is a new type of wireless broadband network. It has the characteristics of self-organization and multi-hop. It has the advantages of both WLAN and ADHoc. It can be divided into three types. One is a single-plane mesh network, the other is a multi-level mesh network, the last is a hybrid network which combines the former two, there are both single-plane, there are also multi-level network level.

\subsection{Classification of Mesh Network Routing Protocols}

According to the route information and maintenance methods, routing protocols can be divided into on-demand, proactive, and hybrid.

According to the algorithm of routing, we can divide into three algorithms: source path, distance vector and reverse route.

\subsection{Types of Routing Protocols for Mesh Networks}

We use more routing protocols in mesh networks: DSR, DSDV, WRP, AODV, AODC several routing protocols.

\section{Optimization of AODC Network Routing Protocol}

\subsection{Optimization Design of Routing Protocol Based on AODC_Improve}

In the process of using mesh network, we often have a high requirement for network delay. Based on this point of departure, this paper proposes a new algorithm based on AODC. The core of the 
algorithm is to calculate the time delay of forwarding from network endpoint according to testing all fixed time in a fixed time. The actual time delay of network endpoints in the previous time range of network endpoints. On the other hand, the network endpoint also has to calculate in a fixed time range, when all packets arriving at the network endpoint within this fixed time range arrive at the network endpoint, all the numbers waiting for the network endpoint to process before the packet, and then calculate how many wait, and then at a fixed time. The number of packets waiting to be processed within the network endpoint and the number of packets waiting to be processed within the last fixed time range are evenly shared with the packets within a fixed time range, so that the estimated time delay at the current network endpoint can be clearly known.

According to the above principle, we get an optimized AODC_Improve, and then we will verify the actual effect of AODC_Improve through data simulation.

\subsection{Data Simulation}

In order to verify the effect of AODC_Improve routing protocol, we compare it with traditional AODC and AODV protocols and do some simulation tests.

The content of the test is divided into two aspects, in a square of $900 \mathrm{~m}$ in size, and then arrange fifty network endpoints in it, and then take an average of 10 to 50, a total of five communication network endpoints to take the simulation test. Packet arrival speed is 5 packets per second, and then according to the $5 \mathrm{~m} / \mathrm{s}$ and $10 \mathrm{~m} / \mathrm{s}$ network terminal movement speed related simulation test, the network to the network terminal time delay and packet loss rate test, packet loss rate is the number of data loss divided by the total amount of data sent.

\subsection{Simulation Content Analysis}

(1) Network terminal to network end time delay

From Fig. 1 and Figure 2, we can clearly find that the network end-to-end delay of the three routing protocols is almost the same when there are few network endpoints. This is mainly because the load of the network is less, the network is better, and the relevant values are almost the same when the number of network endpoints. After the word is over 30, we find that the difference between the three network endpoints is out. This is mainly due to the network load began to increase, AODV routing protocol its routing rule is to choose the minimum number of hops principle, which in turn leads to the network node load is too large, so that each network endpoint waiting queue. The number of packets in Wuzhong is increasing, the number of services waiting to be processed is increasing, and the time becomes very long, so the time delay from network to network is very large. AODC and AODC_Improve routing protocols are routing paths with the shortest latency in the network, which can greatly shorten the network-to-network latency. On the other hand, AODC_Improve takes the related network-to-network latency one step further. Optimization, so you can see clearly from the chart that the network delay of AODC_ Improve is shorter than that of AODC.

Looking at Figure 2, where the rate of movement is $10 \mathrm{~m} / \mathrm{s}$, is $5 \mathrm{~m} / \mathrm{s}$ faster than Figure 1, we can clearly see that the network-to-network latency has increased. The main reason for this is that when the speed increases, the network links are often disconnected and then re-linked, which virtually increases the network-to-network latency. 


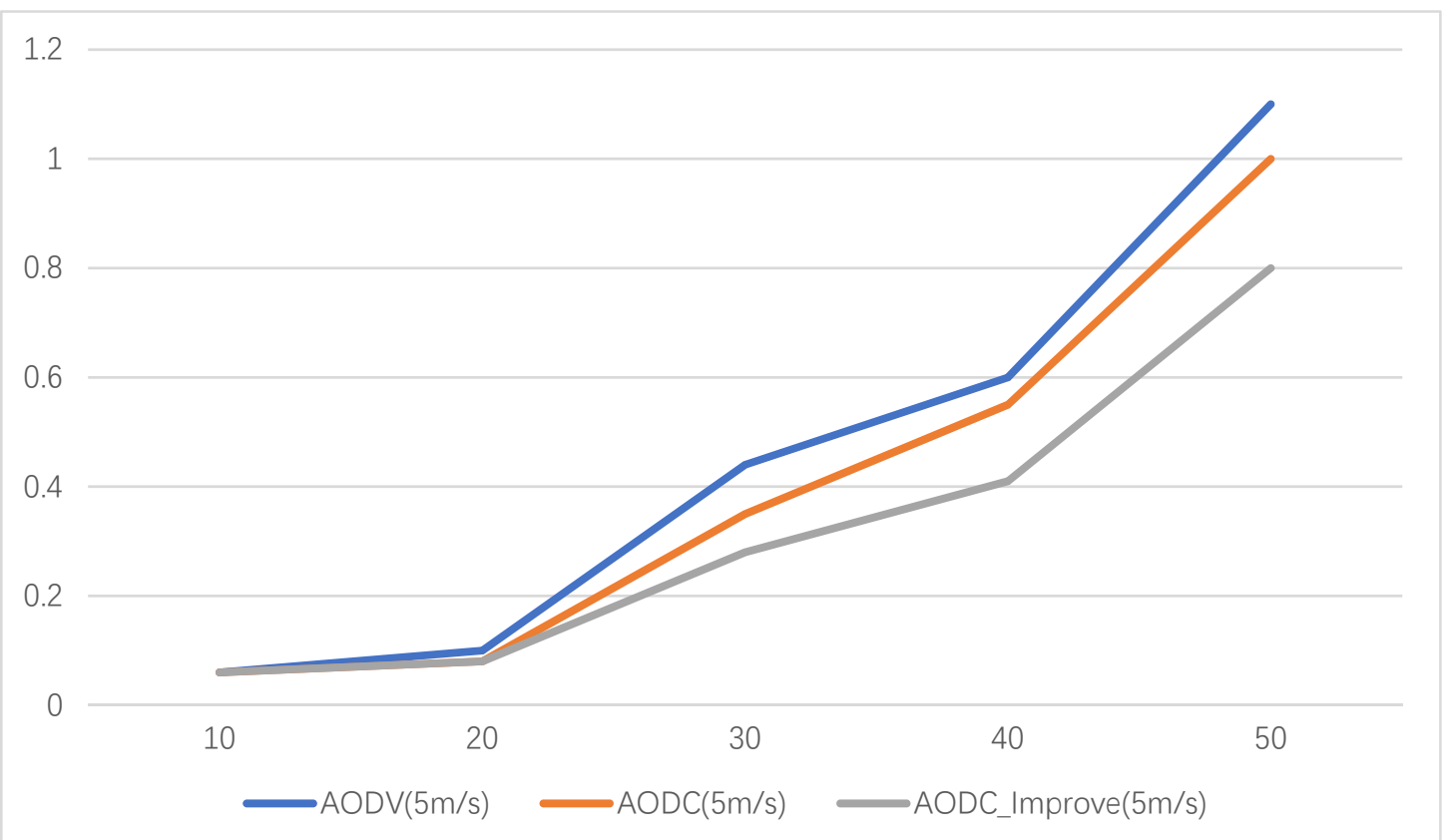

Figure 1. time delay from network end to network end when moving at $5 \mathrm{~m} / \mathrm{s}$ rate. (The horizontal axis represents the number of nodes, and the vertical axis represents the end-to-end delay (S).)

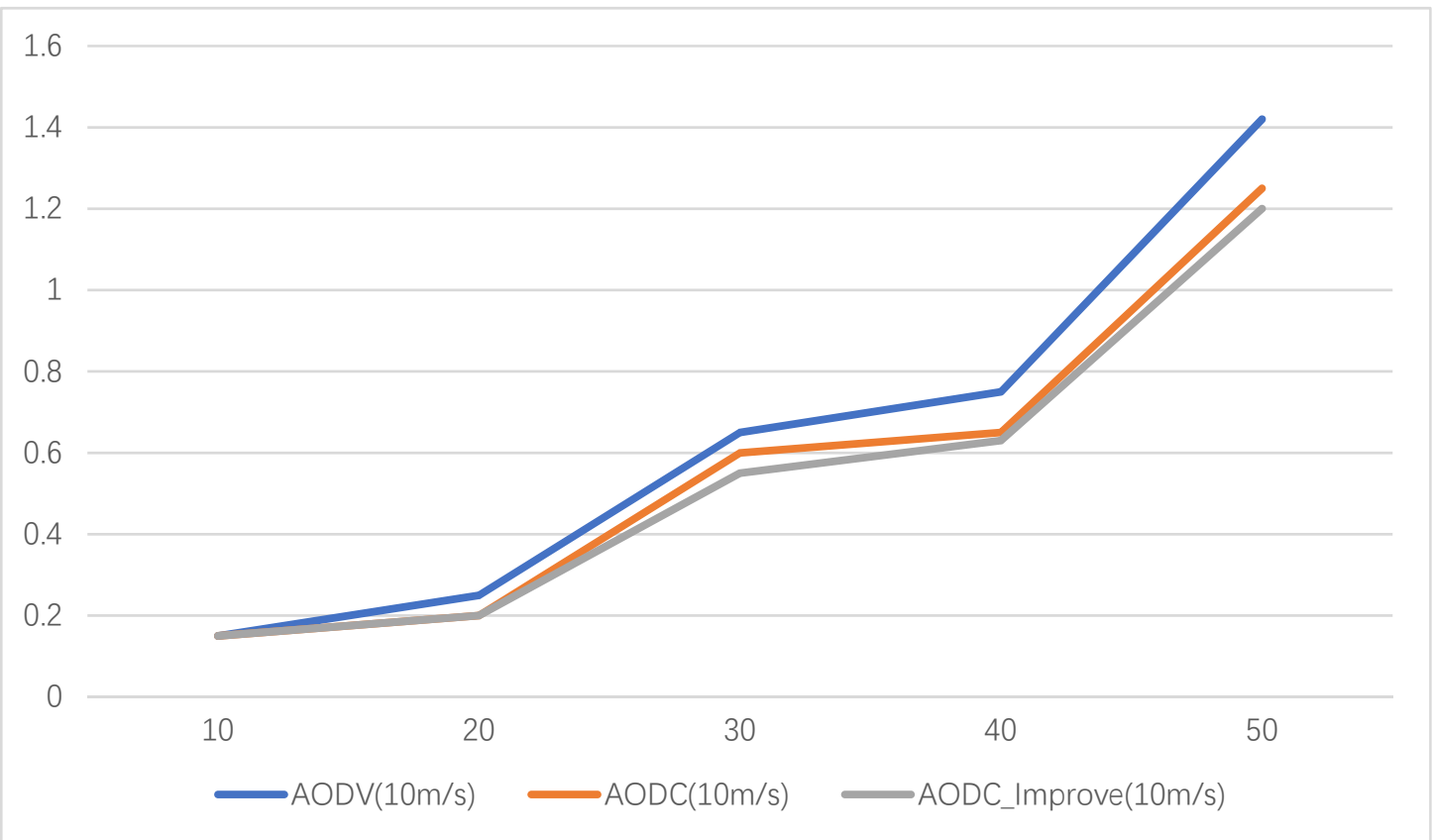

Figure 2. time delay from network end to network end when moving at $10 \mathrm{~m} / \mathrm{s}$ rate.

(2) Packet loss

From Figure 3 and Figure 4, we can find that when there are only ten network terminals, the packet loss of the three routing protocols is similar. This is mainly because there are not many packets in the network environment at that time, and the load of each network endpoint is small, so the problem of packet loss will not occur in general. However, when the number of nodes on the network side increases, the network packet loss of AODC_Improve protocol is much lower than that of AODC and AODV routing protocols, mainly because AODC_Improve protocol is an optimized protocol, which pairs the waiting queue length of nodes on the network end when trying to establish routing connections. Considering the long latency of network endpoints, we can quickly find a path with shorter latency to connect the network. This way, we can avoid finding some network endpoints with smaller latency a few seconds before they can be found, but the delay is larger when the packets are being delivered, so we can avoid data transmission adequately. Loss of packets in the process occurs. 
We can also find that when the rate increases from $5 \mathrm{~m} / \mathrm{s}$ to $10 \mathrm{~m} / \mathrm{s}$, packet loss increases significantly, mainly because when the rate increases, the network link is often disconnected, and then the network is re-linked, which virtually increases the network-to-network delay.

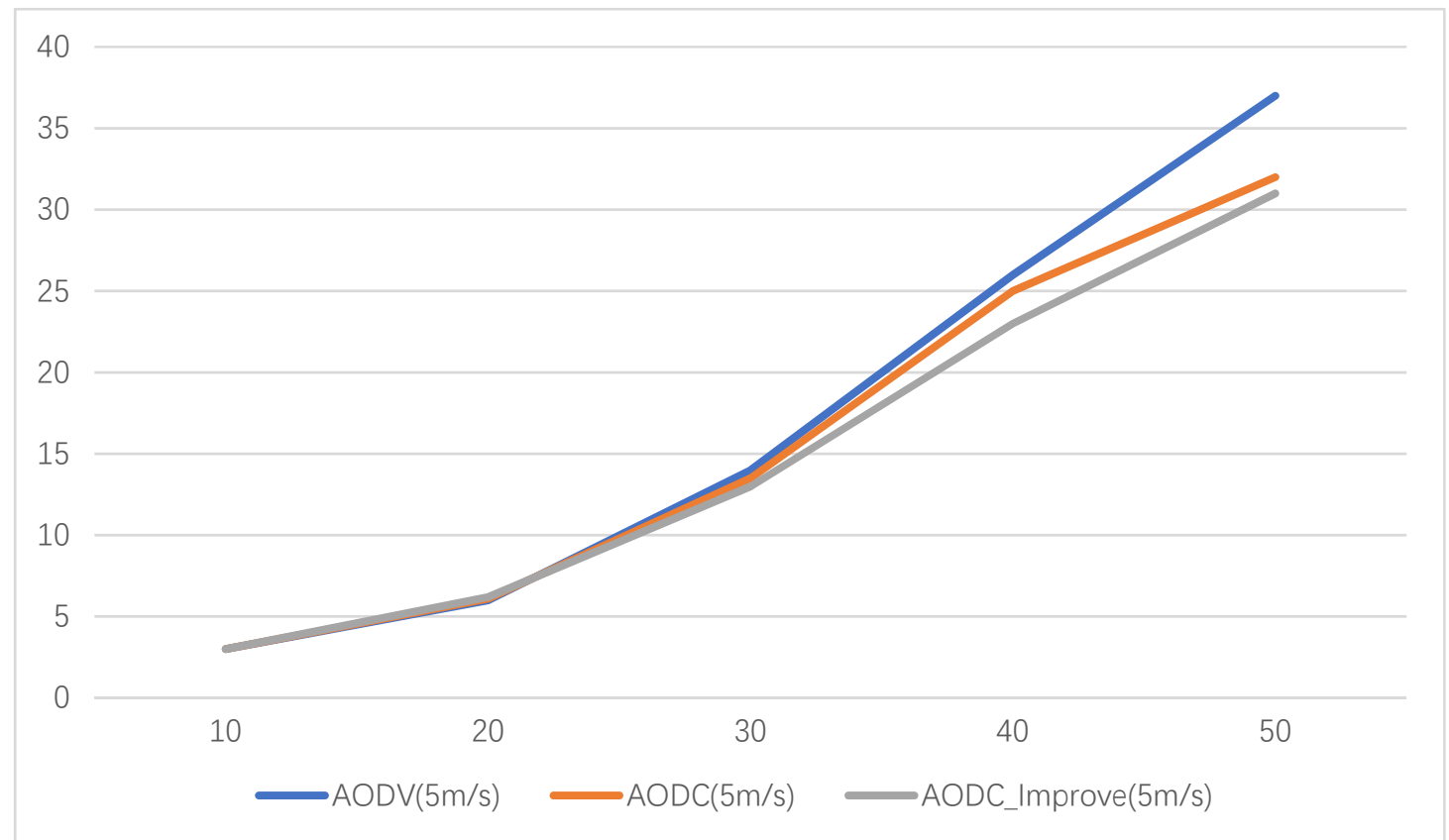

Figure 3. packet loss rate at $5 \mathrm{~m} / \mathrm{s}$ rate

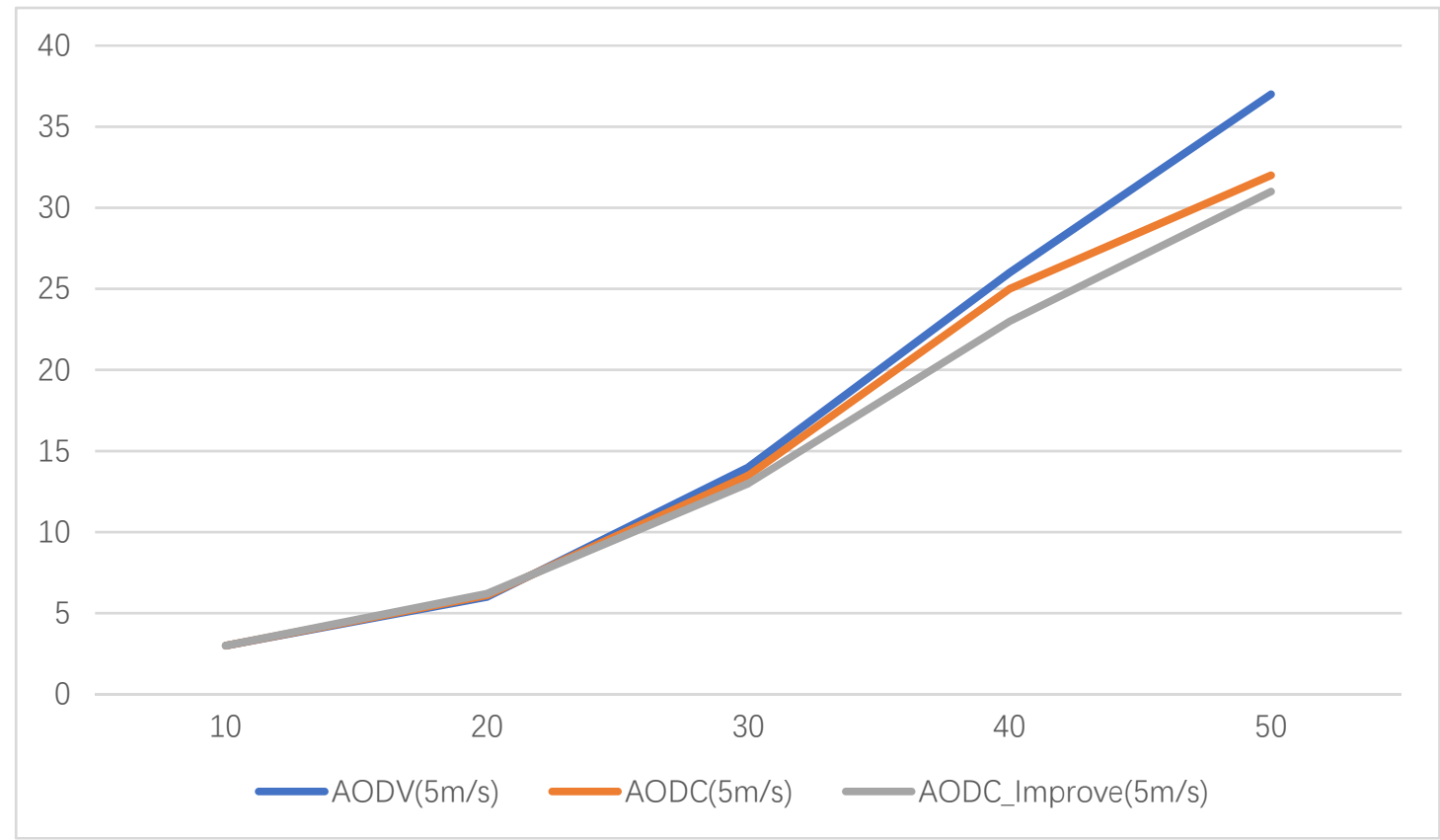

Figure 4. packet loss rate at $10 \mathrm{~m} / \mathrm{s}$ rate

\section{Summary}

This paper proposes an optimized AODC_Improve routing protocol based on AODC. Through simulation test, it can be clearly seen that the node delay and packet loss rate have been significantly improved to improve the use of mesh network. 


\section{Acknowledgments}

Guizhou Province Science and Technology Project (Guizhou Scientific and technological cooperation with LH word [2017] 7070 number).

\section{References}

[1]. Wang Hongxia, Lv Teng. Wireless Mobile Mesh Network Routing Protocol Based on Mobility Prediction [J]. Journal of Electronic Measurement and Instruments, 2018, 32 (04): 165-171.

[2]. Xie Guifang. Routing protocols for dynamic channel assignment in multi-interface and multichannel wireless Mesh networks[J]. Modern Computer (Professional Edition), 2018 (11): 3-6.

[3]. Zhang Weiwei, He Jiafeng, Gao Guowang, Ren Lili, Shen Zhengjing. Joint optimization of routing and channel allocation for hybrid wireless Mesh networks [J].Journal of Jilin University (Engineering Edition), 2018, 48 (01): 268-273.

[4]. Chen Yan, Jiang Nianping. Routing protocol research based on wireless Mesh network[J]. Electronic measurement technology, 2017, 40 (05): 143-147. 